\title{
Meta-analysis of the prognostic role of HE4 expression in cancer patients: clinical insights into interpretation of clinical outcomes
}

This article was published in the following Dove Medical Press journal:

Cancer Management and Research

\author{
Rama Jayaraj' \\ Chellan Kumarasamy ${ }^{2}$ \\ 'College of Health and Human \\ Sciences, Charles Darwin University, \\ Casuarina, NT, Australia; ${ }^{2}$ University \\ of Adelaide, North Terrace Campus, \\ Adelaide, SA, Australia
}

\section{Dear editor}

An article titled, "Prognostic values of HE4 expression in patients with cancer: a metaanalysis", was recently published in the journal, Cancer Management and Research, detailing a study conducted by Dai et al. ${ }^{1}$ This study aims to validate and estimate the effectiveness of HE4 as a prognostic marker in cancer. We commend the authors for the study's focus on all types of cancers, as well as the associated subgroup analysis detailing the results for individual cancer types and population groups. This study is valuable as a guidepost for informing future research on HE4 as a prognostic biomarker to predict the cancer patients' clinical outcomes. However, there are a few points that require to be addressed regarding the study conducted.

\section{Promising biomarker in epithelial cancer diagnosis and prognosis}

It is worth mentioning that HE4 is not only a possible prognostic marker, with recent studies showing that it is also a promising serum and tissue biomarker for diagnosis in lung cancer, ovarian carcinoma, and endometrial cancer, as well as a differential marker for identification of ovarian cancer from benign gynecologic disease., ${ }^{2,3}$ it is emerging as a clinically significant biomarker in ovarian cancer.

\section{Systematic review and meta-analysis guidelines and protocol registration}

Furthermore, the authors have not specified the following of any systematic review and meta-analysis guidelines. These guidelines (such as PRISMA, JBI, and MOOSE) exist to homogenize and standardize systematic reviews and meta-analysis to inform and benefit future research, which is one of the primary impetus behind conducting of a study, which cumulates and analyzes previous existing literature. It is also suggested that the protocols used for performing a systematic review and meta-analysis are registered in the PROSPERO database, which allows peers to replicate the type of study undertaken as well as provide insight on the process behind the study.

\section{Publication bias of the included studies}

It is also worth noting that the evaluation publication bias consists of a few key modules to evaluate the bias of the included studies. Although the Begg's and Eggers' test alongside the funnel plots were used for assessing publication bias in Dai et al's 
study, another key assessment that is worth including is the "Duval and Tweedie's trim and fill" method. This method is an essential module that may be used to impute missing small studies with large effect size to be dispersed equally on either side of the overall effect. ${ }^{4}$

\section{Survival endpoints}

As this study considers disease-free survival (DFS) as an endpoint for its meta-analysis, an evaluation of recurrence rates is also possible as patients that do not fall under DFS would have suffered relapses. This additional analysis is a natural extension of the data already being presented, and might serve to help future research in this field.

\section{The pooled effect size of cancer patients' survival}

Additionally, the pooled HRs for the subgroups of Asian (2.62) and lung cancer (2.31) as well as for DFS of all studies (2.50) are relatively high when compared to other subgroup cohorts and survival endpoints, as they indicate that the probability of death is over twice as likely for the patients expressing HE4 as compared to patients not expressing HE4 in cancer. These marked differences in HR values between different cancers and different populations require further evaluation and analysis.

\section{Threshold effects: correlation between the ranks of effect sizes and the ranks of their variances}

It is also notable that $\chi^{2}$ and $I^{2}$ values may not be sufficient as statistical parameters as they do not consider the threshold effect. In random-effects meta-analysis, it may be useful to also include the $\tau^{2}$ parameter for estimating variance or heterogeneity between the effects for test accuracy, as the $\tau^{2}$ parameter does consider the threshold effect. ${ }^{5}$

These points mentioned above are to address improvements that could benefit Dai et al's study and help elevate its impact on future research. These points should also help guide future similar systematic reviews and meta-analyses.

\section{Disclosure}

The authors declare no conflicts of interest in this communication.

\section{References}

1. Dai C, Zheng Y, LiY, et al. Prognostic values of HE4 expression in patients with cancer: a meta-analysis. Cancer Manag Res. 2018;10:4491-4500.

2. Bie Y, Zhang Z. Diagnostic value of serum HE4 in endometrial cancer: a meta-analysis. World J Surg Oncol. 2014;12(1):169.

3. Wu L, Dai ZY, Qian YH, Shi Y, Liu FJ, Yang C. Diagnostic value of serum human epididymis protein 4 (HE4) in ovarian carcinoma: a systematic review and meta-analysis. Int J Gynecol Canc. 2012;22(7):1106-1112.

4. Duval S, Tweedie R. A nonparametric "trim and fill" method of accounting for publication bias in meta-analysis. J Am Stat Assoc. 2000;95(449):89-98.

5. Jayaraj R, Kumarasamy C, Ramalingam S, Devi A. Systematic review and meta-analysis of risk-reductive dental strategies for medication related osteonecrosis of the jaw among cancer patients: approaches and strategies. Oral Oncol. 2018;86:312-313. 


\section{Authors' reply}

\section{Cong Dai,' Yi Zheng,' Tian Tian,' Zhijun Dai,' Jun Lyu ${ }^{2}$ \\ 'Department of Oncology, Second Affiliated Hospital of Xi'an jiaotong University, Xi'an, Shaanxi 7I 0004, China; 'Jun Lyu, Clinical Research Center, First Affiliated Hospital of Xi'an Jiaotong University, Xi'an 710004, China}

Correspondence: Zhijun Dai

Department of Oncology, Second Affiliated Hospital of Xi'an Jiaotong

University, Xi'an 710004, China

Email dzj09।I@I26.com

Jun Lyu

Clinical Research Center, First Affiliated Hospital of Xi'an Jiaotong

University, Xi'an 710004, China

Email lujun2006@xjtu.edu.cn

\section{Dear editor}

We thank Dr Rama Jayaraj and Chellan Kumarasamy for their interest and comments. They came up with several valuable points that improved our meta-analysis. ${ }^{1}$

As they mentioned, recent studies ${ }^{2,3}$ showed that HE4 was a promising serum and tissue biomarker for diagnosis in lung cancer, ovarian carcinoma, and endometrial cancer, but its prognostic value remains controversial. Therefore, this is why we did this study to accurately assess the prognostic value of HE4 expression in cancer patients.

Our meta-analysis is based on Preferred Reporting Items for Systematic Reviews and Meta-Analysis (PRISMA) guidelines. ${ }^{4}$ However, the review protocol was not registered in the PROSPERO database, which is an oversight of our work.

In terms of publication bias of the included studies, we think that the use of Begg's and Eggers' test alongside the funnel plots was enough to assess the publication bias. What's more important, the "Duval and Tweedie's trim and fill" method $^{5}$ was currently controversial in assessing publication bias. So we did not use this method at that time. Now we can provide the result here. The combined HR and 95\% CI of filled meta-analysis is 1.645 and 1.352-2.002 (randomeffects model) (Figure 1).

We would like to further explore some subgroups or more survival endpoints, such as Asian, lung cancer, disease-free survival, and the other you mentioned. But due to the lack of relevant researches, it cannot be achieved. So, more studies are needed to clarify these questions.

As mentioned by Dr Rama Jayaraj and Chellan Kumarasamy, in random-effects meta-analysis, it was more accurate to use $\tau^{2}$ parameter for estimating heterogeneity, when compared with $\chi^{2}$ and $I^{2}$ values. Nevertheless, there is no difference between the two results in our study.

Based on the above, despite those limitations mentioned above, the final conclusion was still accurate. Overall, we really appreciate the comments from Dr Rama Jayaraj and Chellan Kumarasamy.

\section{Disclosure}

The authors report no conflicts of interest in this communication.

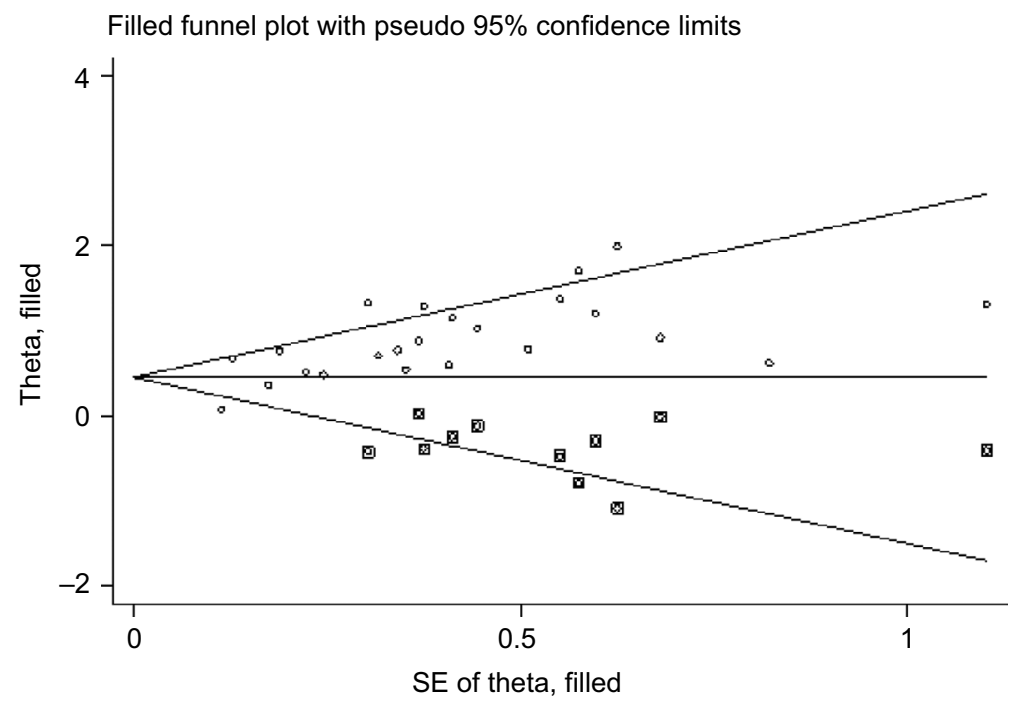

Figure I Filled funnel plots of publication bias for all of the included studies reported with overall survival. Abbreviation: SE, standard error. 


\section{References}

1. Dai C, Zheng Y, LiY, et al. Prognostic values of HE4 expression in patients with cancer: a meta-analysis. Cancer Manag Res. 2018;10:4491-4500.

2. Cheng D, Sun Y, He H. The diagnostic accuracy of HE4 in lung cancer: a meta-analysis. Dis Markers. 2015;2015(6):1-7.

3. Dayyani F, Uhlig S, Colson B, et al. Diagnostic performance of risk of ovarian malignancy algorithm against CA125 and HE4 in connection with ovarian cancer. Int J Gynecol Canc. 2016;26(9):1586-1593.
4. Moher D, Liberati A, Tetzlaff J, Altman DG; PRISMA Group. Preferred reporting items for systematic reviews and meta-analyses: the PRISMA statement. Open Med. 2009;3(3):e123-130.

5. Duval S, Tweedie R. A nonparametric "trim and fill" method of accounting for publication bias in meta-analysis. J Am Stat Assoc. 2000;95(449):89-98.

Dove Medical Press encourages responsible, free and frank academic debate. The content of the Cancer Management and Research 'letters to the editor' section does not necessarily represent the views of Dove Medical Press, its officers, agents, employees, related entities or the Cancer Management and Research editors. While all reasonable steps have been taken to confirm the content of each letter, Dove Medical Press accepts no liability in respect of the content of any letter, nor is it responsible for the content and accuracy of any letter to the editor.

\section{Publish your work in this journal}

Cancer Management and Research is an international, peer-reviewed open access journal focusing on cancer research and the optimal use of preventative and integrated treatment interventions to achieve improved outcomes, enhanced survival and quality of life for the cancer patient. The manuscript management system is completely online and includes a very quick and fair peer-review system, which is all easy to use. Visit http://www.dovepress.com/testimonials.php to read real quotes from published authors. 